\title{
YACÓN COMO PLANTA PROMISORIA EN EL MANEJO DE ENFERMEDADES
}

Lyda Caballero Méndez ${ }^{1}$, Ana María Colonia Pineda ${ }^{2}$

\section{Resumen}

Una de las grandes preocupaciones en la medicina humana es el aumento significativo del número de pacientes con enfermedades que disminuyen la expectativa de vida y afectan su calidad, como es el caso de la diabetes, dislipidemias y cáncer de colón, entre otras. Los tratamientos convencionales para estas enfermedades en algunos casos no son exitosos, lo que conlleva a empeorar la condición clínica o incluso, la muerte del paciente. Por lo anterior, surge la necesidad de buscar otras opciones terapéuticas que conlleven a mejorar la condición clínica, que sean de fácil adherencia, y su consumo sea seguro y accesible para la población. Por tanto se sugiere el yacón como una planta medicinal con un amplio historial de consumo seguro y una gran evidencia científica que respalda su uso. Sus propiedades biológicas únicas, tales como antimicrobianas, antifúngicas, antioxidantes, inmunomoduladoras, prebióticas y anticancerígenas, entre otras, hacen de esta planta, una promesa para la medicina tradicional humana.

1 Magíster en Biología Molecular y Biotecnología (c). Docente Universidad Tecnológica de Pereira. Colombia. lydaccm_27@utp.edu.co

${ }^{2}$ Magíster en Bromatología (c). Docente de la Universidad Tecnológica de Pereira.

Colombia.Anamaria.colonia@utp.edu.co 


\section{YACÓN AS A PROMISING PLANT IN THE TREATMENT OF DISEASES}

Lyda Caballero Méndez, Ana María Colonia Pineda

\section{Abstract}

One of the major concerns in human medicine is the significant increase in the amount of patients with diseases that reduce life expectancy and affect its quality, such as diabetes, dyslipidemias and colon cancer, among others. The conventional treatments for these diseases some times are not successful, which lead the patient to a worst clinical condition or even cause death. Due to the above, there is a need of other therapeutic options that lead to improve the clinical condition, which can be easily performed, and its consumption is safe and accessible to the population. Therefore, Yacón is suggested as a medicinal plant that has a long history of safe consumption and a great scientific evidence to support its use. Its unique biological properties, such as antimicrobial, antifungal, antioxidant, immunomodulatory, prebiotic and anticancer, among others, make this plant a promise for traditional human medicine. 


\section{YACON COMO PLANTA PROMISSORA NO MANEJO DE DOENÇAS}

Lyda Caballero Méndez, Ana María Colonia Pineda

\section{Resumo}

Uma das principais preocupações da medicina humana é o aumento significativo do número de pacientes com doenças que reduzem a expectativa de vida e afetam sua qualidade, como diabetes, dislipidemias e câncer de cólon, entre outros. Tratamentos convencionais para essas doenças, em alguns casos, não são bem-sucedidos, o que leva a piorar a condição clínica ou até mesmo a morte do paciente. Portanto, existe a necessidade de buscar outras opções terapêuticas que levem à melhora da condição clínica que sejam de fácil adesão, e de consumo seguro e acessível à população. Portanto, yacon é sugerido como uma planta medicinal com uma longa história de consumo seguro e uma grande evidência científica para apoiar seu uso. Suas propriedades biológicas únicas como planta antimicrobiana, antifúngica, antioxidante, imunomoduladora, prebiótica e anticâncer, entre outros, fazem desta planta uma promessa para a medicina humana tradicional. 


\section{Introducción}

Las tendencias mundiales de la alimentación en los últimos años indican un interés de los consumidores a ingerir ciertos alimentos que además de contener nutrientes, contengan sustancias fisiológicamente activas que cumplan, al igual que los nutrientes esenciales, una función beneficiosa en la reducción de ciertas enfermedades (1). Numerosos estudios en nutrición humana, demuestran una estrecha correlación entre el consumo de frutas y verduras y la menor incidencia de enfermedades crónico degenerativas, debido a su bajo contenido en colesterol y a la presencia de vitaminas, fibras, antioxidantes naturales y minerales $(2,3)$.

Una de las herramientas terapéuticas usadas desde tiempo atrás y con gran auge en la actualidad, es el uso de las propiedades medicinales de algunas plantas. Sus beneficios y acciones terapéuticas estarían relacionadas con algunas moléculas y a la presencia de antioxidantes con capacidad para captar radicales libres derivados del oxígeno (ROS) que están involucrados con el estrés oxidativo, responsable de la patogénesis de muchas enfermedades humanas, como arterioesclerosis, artritis, demencia y cáncer, etc. (4). El yacón cuyo nombre científico es Smallanthus sonchifolius (Poepp.) H.Rob., es considerado una planta medicinal utilizada como un alimento funcional, debido a las grandes cantidades de fructanos como inulina $y$ fructooligosacáridos (FOS) que constituyen la reserva de la planta $(5,6,7,8)$.

Sus raíces son empleadas como suplementos en la dieta, al acumular FOS en lugar de almidón, lo que conlleva a mantener estables los niveles de glucosa sanguíneos; a su vez, a esta planta se le atribuyen propiedades prebióticas, antimicrobianas, antifúngicas, antioxidantes y efectos anticáncer, entre otras (9-11). Por lo anterior, el yacón es considerado una planta medicinal y un alimento ideal para tratar ciertas enfermedades.

Esta revisión tuvo como objetivo actualizar la información sobre el yacón y su uso como planta medicinal, se centró en la investigación basada en el uso del yacón aplicado al campo de la medicina humana como herramienta terapéutica para tratar enfermedades que deterioran la calidad de vida del paciente. Se realizó una búsqueda bibliográfica en bases de datos como Pubmed, Science direct, Scopus, Lilacs, teniendo en cuenta artículos relacionados con el yacón, con información relevante y reciente, concerniente a la prevención y tratamiento de enfermedades humanas. Se excluyeron aquellos artículos relacionados con su biología molecular, ya que no era la finalidad de la revisión.

\section{Características del yacón}

El yacón es una planta perenne, herbácea, considerada como hierba medicinal perteneciente a la familia Asteraceae (13), en otros países es conocida con el nombre de llaqon, llacum, llacuma, yacumpi, aricuma, chicama, jiquima y jiquimilla, poire de terre y fresa de yacón $(15,16)$.

Es una especie originaria de los Andes, cultivada para el consumo de sus raíces, desde Venezuela hasta el noreste argentino, incluyendo Bolivia y Perú $(17,18)$. Recientemente fue introdu- 
cida con éxito en el mercado europeo y en mercados de Nueva Zelanda, Japón, Estados Unidos y Brasil, donde se desarrolla una industria creciente del cultivo, apuntando a sus propiedades dietéticas y medicinales (19-21). Es hasta 1980 que esta especie despertó el interés por parte de la comunidad científica y de los consumidores, debido al considerable crecimiento en la tasa de pacientes con enfermedades crónicas relacionadas con la dieta, especialmente en países industrializados (22).

Es una planta de 1,5 a $3 \mathrm{~m}$ de altura, cultivada en climas tropicales y subtropicales entre los $1300 \mathrm{msnm}$ hasta 3500 msnm, sus hojas son pinnatífidas en la base de los tallos y triangulares en la parte apical, con flores que aparecen en racimos terminales $(23,24)$. Esta planta se siembra en cualquier época del año y no necesita de mayores cuidados, alcanza su maduración a los seis o siete meses y la parte aérea muere después de florecer (8). Con respecto a sus raíces, son de sabor dulce y agradable, las cuales pueden consumirse crudas o soleadas, pueden medir entre $5-25 \mathrm{~cm}$ y se pueden presentar entre 5-40 raíces por planta (25); pueden ser blancas crema, blancas con estrías púrpura, moradas, rosadas o amarillas (19).

Estas raíces pueden ser de dos tipos: las fibrosas muy delgadas, cuyas funciones son fijar la planta al suelo y absorber los nutrientes y el agua, y las raíces reservantes o tuberculosas, que son engrosadas y fusiformes, cuyo peso puede variar entre $2-4 \mathrm{~kg}$ y son las encargadas de almacenar oligofructanos y azúcares simples $(10,22)$.
No tiene problemas con plagas o enfermedades, debido al contenido en sus moléculas como sesquiterpenos (26). En cuanto a su biología reproductiva predomina la propagación clonal, mientras que a nivel de la reproducción sexual se reporta reducida producción de flores y baja viabilidad de semillas. La estructura cromosómica de su cultivo es octoploide $(6 \mathrm{~A}+2 \mathrm{~B}$ con $2 \mathrm{n}=58)$, probablemente como resultado del cruce inespecífico entre S. macroscypus y S. riparius (27). En países como Ecuador, Bolivia y Perú se mantienen bancos de genes de esta planta (14) . El yacón es considerado una planta medicinal y un alimento funcional, sus tubérculos y hojas son utilizados en la industria alimentaria como fuente para la producción de edulcorantes, mermeladas, dulces, yogurt, rodajas secas, pasteles, té y jarabes naturales con amplias aplicaciones en el campo de la medicina humana (28).

\section{Composición química de yacón}

Con respecto al contenido hídrico se conoce que entre el 83 y $90 \%$ del peso fresco es agua y los carbohidratos representan alrededor del $90 \%$ del peso seco en sus raíces $(10,27,29)$. Su raíz reservante se caracteriza por su alto contenido de carbohidratos no digeribles representados por los oligofructanos que se encuentran en un rango entre $50-80 \mathrm{~g} / \mathrm{kg}$, azúcares libres entre $18-31 \mathrm{~g} / \mathrm{kg}$ (glucosa 2,3-5,9\%, fructosa 3,9-21,1\% y sacarosa $10-19 \%)$; contenido proteico entre 2,7$4,9 \mathrm{~g} / \mathrm{kg}$ y grasas entre $112-464 \mathrm{mg} / \mathrm{kg}$, por lo que su valor energético se calcula entre 148-224 Kcal/kg (30).

Tiene un contenido total de fibra alrededor de $45 \mathrm{~g} / 100 \mathrm{~g}$ de muestra, de los 
cuales $35 \mathrm{~g}$ son fructanos de tipo inulina y los $10 \mathrm{~g}$ restantes son polisacáridos celulósicos y no celulósicos (8). Esta raíz es considerada un alimento funcional, debido a la gran cantidad de carbohidratos o fructanos que almacena en forma de inulina y carbohidratos de tipo fructosa, unidos por enlaces glucosídicos $\beta(1 \rightarrow 2)$ con una sacarosa terminal, formando fructooligosacáridos (FOS, polímeros de fructosa), que son capaces de resistir la hidrólisis de enzimas del tracto superior del sistema gastrointestinal humano, dándole bajo contenido calórico (19, $21,22,31,32)$. Los FOS representan en el yacón, el $80-90 \%$ de su peso seco (33), sus principales productos del metabolismo son los ácidos grasos de cadena corta, como el butirato, acetato y propionato $(26,31,34)$.

Además, las raíces de yacón son ricas en compuestos fenólicos, principalmente ácido clorogénico, ácido ferúlico y ácido cafeíco en una concentración de 43,38 $\mathrm{mg} / \mathrm{Kg}$ peso fresco, $21,22 \mathrm{mg} / \mathrm{Kg}$ peso fresco y 7,28 mg/Kg peso fresco, respectivamente, a los que se les atribuye su capacidad antioxidante (35). Sin embargo, el contenido de compuestos polifenólicos puede variar dependiendo del genotipo estudiado (19). De las hojas de yacón han sido aislados compuestos de naturaleza sesquiterpénica, flavonoides y trazas de FOS; del extracto metanólico foliar se aisló ácido kaurénico y compuestos relacionados, cuatro lactonas sesquiterpénicas del tipo melanpolido con actividad antifúngica y antibiótica (enidrina, uvedalina, sonchifolina y polimatina B) y cuatro gliceroglicolípidos con propiedades insecticidas; el compuesto sesquiterpénico llamado enidrina muestra actividad antiinflamatoria, antioxidante, antifúngica y antimicrobiana, y es también un agente antidiabético patentado como componente de formulaciones farmacéuticas (36).

Con respecto al contenido de flavonoides presentes en las hojas de yacón, se encontró 5,7-dihidroxi-4>-metoxiflavonol, 5,7,3>-trihidroxi-4>-metoxiflavonol, 5-hidroxi-4>metoxi-7-0-glicosilflavona y 7,4>-dihidroxi-3,5>-dimetoxiflavona, los cuales no mostraron una toxicidad significativa (37). De igual manera, se han detectado otros elementos minerales como potasio, calcio, magnesio, zinc, fósforo, sodio, hierro y manganeso; aminoácidos esenciales como triptófano, valina y treonina; aceites esenciales como $\beta$-pineno, cariofileno y $\gamma$-cadideno; vitaminas como retinol, caroteno, tiamina, riboflavina, niacina y ácido ascórbico (15) y alrededor de 25 compuestos fenólicos diferentes. Por tanto es preciso afirmar que el yacón es un alimento funcional y una planta prometedora en el tratamiento de enfermedades (29).

\section{Beneficios para la salud humana}

La expansión global de la producción y comercialización del yacón se dio posterior a estudios que relacionaban el consumo de esta planta con beneficios para la salud humana (38). En la medicina tradicional, las raíces de yacón tienen un largo historial de uso seguro y benéfico, gracias a sus potenciales propiedades promotoras de la salud, entre las que se incluye la actividad antioxidante que le confiere los compuestos fenólicos, la reducción en los niveles de glucosa sanguínea atribuido al perfil de carbohidratos, propiedades prebióticas, proporcionadas por los azúcares de sus raíces que 
estimulan el crecimiento y desarrollo de la flora intestinal, capacidad de inhibir el crecimiento bacteriano y fúngico, entre otras $(29,38,40)$. La infusión de las raíces es consumida por personas con trastornos diabéticos, digestivos y renales para mejorar su cuadro clínico $(21,41)$.

También se le ha atribuido que puede prevenir y mejorar el estreñimiento, reducir los niveles de lípidos y glucosa en sangre de animales y sujetos diabéticos (31), lo cual fue probado por Baroni y Col., quienes usaron extractos de yacón para reducir los niveles de glucosa en sangre de animales diabéticos, a su vez demostraron que la fracción hidroetanólica del extracto fue capaz de reducir el contenido de glucógeno en el hígado y músculo esquelético, restaurando la actividad de la glucosa 6-fosfato deshidrogenasa en ratas diabéticas tipo 1 (42).

Otros estudios han demostrado que el extracto metanólico, butanólico y clorofórmico, probado en un modelo de diabetes tipo 1 inducido por estreptozotocina, aumentó los niveles de insulina en plasma, conllevando a la reducción de la glucosa en la sangre (43).

Esta capacidad hipoglicemiante es corroborada por Aybar y col., quienes demostraron que la administración del extracto de hojas de yacón en forma aguda (vía intraperitoneal o por sonda orogástrica) en ratas normales, produce una disminución de los niveles de glucosa sérica y un descenso marcado de la glicemia, por un aumento de insulina plasmática en ratas diabéticas tipo 2 (inducidas por estreptozotocina a dosis bajas), posterior a la administración crónica de té de hojas de yacón por 30 días $(30,44,45)$.
Estudios adicionales han demostrado que los compuestos fenólicos, específicamente los derivados del ácido clorogénico como los ácidos altraricos aislados de hojas y raíces de yacón, tienen la potente capacidad de inhibir la enzima a-glucosidasa, responsable de la degradación del glucógeno a glucosa en los lisosomas, postulando su uso como un potente hipoglicemiante oral (20). De igual manera, el ácido clorogénico demostró modificar las concentraciones plasmáticas y hepáticas de colesterol y triglicéridos (22). Estudios afirman que el consumo de esta planta está directamente relacionado con la prevención del cáncer de colon, ya que el crecimiento de bacterias como Bifidus y Lactobacillus se ve favorecido por el contenido de FOS e inulina, lo que conlleva a modificar y equilibrar la microflora del tracto gastrointestinal e inhibir el establecimiento de bacterias patógenas, promoviendo la absorción de minerales y metabolitos a nivel del tracto gastrointestinal $(5,21$, $22,46,47,48)$. Por tanto, el consumo de yacón mejora el número de microorganismos probióticos, demostrando su gran potencial como alimento prebiótico en la industria alimentaria (49).

Así mismo, el contenido de fructanos tiene propiedades inmunomoduladoras. Estas moléculas no son hidrolizadas por las enzimas del tracto digestivo humano, por tanto son consideradas fibra dietética, ya que al pasar por el tracto gastrointestinal conservan su estructura, estas moléculas son fermentadas por las micro bacterias propias del intestino, transformándolas en ácidos grasos de cadena corta como el acetato, propionato, butirato, ácido láctico, dióxido de 
que sirve como combustible para los enterocitos, desintoxica la mucosa intestinal de compuestos carcinogénicos (34), mientras que el acetato y el propionato, penetran en la sangre portal y pueden influir en el metabolismo de carbohidratos y lípidos (31).

Además como prebióticos, los alimentos ricos en oligofructanos favorecen la evacuación intestinal y reducen el riesgo de osteropororsis, mejorando la biodisponibilidad de calcio y previniendo la obesidad (22). El consumo de FOS cambia la histología intestinal, al aumentar la profundidad y la cantidad de criptas totales y bifurcadas $(34,50)$; además puede reducir el número y la multiplicidad de focos de criptas aberrantes (FCA), el número de adenocarcinomas invasivos y la tasa de proliferación celular en tumores (34). Así mismo, el tratamiento oral con yacón redujo notablemente el tiempo de tránsito colónico, aumentó la frecuencia de defecación y la sensación de saciedad en personas obesas, dislipidemicas y premenopaúsicas (21). La ingesta diaria de jarabe de yacón está relacionada con la disminución del peso corporal, la circunferencia de la cintura y el índice de masa corporal, demostrando su importante papel en el manejo de la obesidad (39).

El extracto hidroalcohólico de las hojas de yacón contiene flavonoides que pueden actuar modulando la respuesta de linfocitos $\mathrm{T}$, linfocitos $\mathrm{B}$, macrófagos, monocitos, células NK, basófilos, mastocitos, neutrófilos, eosinófilos y plaquetas; se ha demostrado que los flavonoles del yacón como la quercetina, kamferol y la miricetina actúan como potentes inhibidores en la liberación de histamina en células tipo mastocitos, un mediador implicado en procesos alérgicos en modelo de ratón (37).

De igual manera, se ha demostrado que los FOS contenidos en el yacón tienen la capacidad de aumentar el porcentaje de fagocitosis a nivel de macrófagos, por tanto, esta planta tiene un efecto directo sobre la respuesta inmune del individuo (32). El consumo de FOS estimula la respuesta inmune local por medio de la producción de IgA secretora, considerada la primera línea de defensa contra patógenos microbianos que ingresan por mucosa intestinal. Así mismo, aumenta la producción de IL-10 que es producida por células $T$, teniendo un efecto sobre macrófagos, células dendríticas y células epiteliales que promueven la eliminación del patógeno y la activación de una respuesta inmune específica y estimula la producción de IL-4 citoquina involucrada en modular el equilibrio entre la respuesta pro/anti inflamatoria (50).

Por otro lado, estudios sugieren que las hojas de yacón han sido utilizadas para tratar los desordenes de la piel y del riñón (20), como rejuvenecedor de la piel y como herramienta para retardar el envejecimiento. $(10,16,51)$.

Por lo anteriormente expuesto, el yacón puede considerarse un cultivo interesante con gran valor a nivel económico, industrial, farmacéutico y ambiental.

\section{Capacidad antioxidante del yacón}

El estrés oxidativo se define como una situación en la que existe tanto, un aumento en la velocidad de generación de las especies reactivas de oxígeno (EROs), entre las que se incluye la sobreproduc- 
ción de radicales libres como el anión superóxido $(\mathrm{O} 2)$, el peróxido de hidrógeno (H2O2), el radical oxidrilo (OH-), el óxido nítrico (NO) y peróxidos (ROO-), así como una disminución en los sistemas de defensa, lo anterior conlleva al daño de diversas estructuras celulares $(16,52)$. Esta situación se ha relacionado con la patogénesis y evolución de la mayoría de las enfermedades crónicas, entre las que se encuentran patológicas cardiovasculares, enfermedades neurodegenerativas y diabetes $(33,51)$.

Para contrarrestar el daño se emplean moléculas conocidas como antioxidantes, que actúan antes o durante una reacción en cadena de los radicales libres, ya sea en la etapa de iniciación, propagación, terminación, descomposición o en la subsecuente oxidación de los productos. Estos antioxidantes protegen las membranas celulares contra el daño realizado por los radicales libres (51). Los flavonoides aislados del yacón han demostrado tener una gran actividad biológica, principalmente como antioxidantes, en esta planta se encuentra en mayor cantidad el 5,7-dihidroxi-4'-metoxiflavonol y el 5,7,3 trihidroxi-4'-metoxiflavonol, estas moléculas tienen la capacidad de actuar, protegiendo las membranas de los microsomas hepáticos, penetrando los espacios acuosos y lipídicos de dichas membranas, para neutralizar el radical peróxido producido por el H2O2 (37).

De igual manera, la actividad antioxidante ha sido atribuida a la alta concentración de compuestos fenólicos presentes en hojas y raíces de yacón, tales como el ácido cafeíco, ácido ferúlico y el ácido clorogénico y sus derivados, entre los que se encuentran los ácidos 3,4-dicafeoilquínico, 3,5-dicafeoilquínico, 4,5-dicafeoilquínico, 2,3,5-tricafeoilaltrárico, 2,4,5- tricafeoilaltrárico (20).

Finalmente, se han reportado efectos hepatoprotectores, gracias a su contenido en fenoles, flavonoides, terpenoides y otros metabolitos secundarios con actividad antioxidante que pueden convertir a los radicales libres en compuestos más estables (16).

\section{Contraindicaciones en el consumo de yacón}

Aunque varias actividades biológicas han sido acuñadas a los extractos de yacón, estudios recientes demuestran que el consumo crónico de extractos de yacón causan toxicidad y daño renal en ratas, debido al contenido de lactonas, serquiterpenos y derivados del ácido clorogénico (53). De igual manera, Mayta y col demostraron que el consumo de grandes cantidades de raíces puede ocasionar eventualmente un efecto laxante, debido al alto contenido de oligofructanos (30). Finalmente, ha sido reportado un efecto adverso grave en una mujer de 55 años relacionado con el consumo de yacón, este efecto fue un caso de reacción anafiláctica posterior a su ingestión confirmada por pruebas de sensibilidad (15).

\section{Perspectivas futuras}

Se ha demostrado que el yacón es una planta medicinal con un amplio historial de beneficios que pueden ser utilizados para mejorar la calidad de vida de 
pacientes con enfermedades crónico degenerativas. Esta planta puede significar una herramienta terapéutica con mínimos efectos secundarios, fácilmente cultivable y con grandes beneficios para la salud humana. Se puede afirmar que en un futuro esta planta pueda utilizarse en conjunto con los medicamentos actuales para tratar enfermedades como la diabetes, dislipidemias, cáncer, infecciones bacterianas-fúngicas y para prevenir el envejecimiento. Por tanto, el consumo

\section{Referencias}

1. Castañeda BC, Llica ER, Vásquez LI. Evaluación de la capacidad antioxidante de siete plantas medicinales peruanas. Horizonte Médico; 2008 8(1).

2. Gutiérrez JB. Calidad de vida, alimentos y salud humana: fundamentos científicos. Ediciones Díaz de Santos; 2012.

3. Gil Á, et al. Tratado de nutrición. Médica Panamericana; 2010. 9(2):1-3.

4. Arnao I, et al. Evaluación de la capacidad antioxidante de los extractos acuosos de la raíz y las hojas de Smallanthus sonchifolius (yacón). Revista de la Sociedad Química del Perú, 2012; 78(2):120-125.

5. Delgado GTC et al. Yacon (Smallanthus sonchifolius)-derived fructooligosaccharides improves the immune parameters in the mouse. Nutrition Research. 2012;32(11): 884-892.

6. Gordillo GC. Efecto hipoglicemiante del extracto acuoso de las hojas de Smallanthus sonchisfolius (yacón) en pacientes con diabetes mellitus tipo 2. 2009. de yacón constituye una gran promesa que puede mejorar la calidad de vida del paciente.

\section{Conflicto de intereses}

Los autores declaramos que no tenemos ningún conflicto de interés.

\section{Financiación}

Ninguna.

7. Flores D. Uso Histórico: Yacón Smallanthus sonchifolius (Poepp.) H. Rob. 2010.

8. Castro A et al. Dietary fiber, fructooligosaccharides, and physicochemical properties of homogenized aqueous suspensions of yacon (Smallanthus sonchifolius). Food research international. 2013; 50(1): 392-400.

9. Manrique I, M. Hermann. El potencial del yacón en la salud y la nutrición. En XI Congreso Internacional de Cultivos Andinos. Cochabamba. 2003.

10. Valderrama, Manrique I. El yacón: fundamentos para el aprovechamiento de un recurso promisorio. En Seminario S.C, International Potato Center. 2003.

11. De Andrade EF et al. Phenolic profile and antioxidant activity of extracts of leaves and flowers of yacon (Smallanthus sonchifolius). Industrial Crops and Products. 2014; 62: 499-506.

12. Kamimura, G.K.F., Isolamento, purificação e caracterização da peroxidase de yacon (Smallanthus sonchifolius). 2006. 
13. Castro A, et al. Investigación de metabolitos secundarios en plantas medicinales con efecto hipoglicemiante y determinación del cromo como factor de tolerancia a la glucosa. Ciencia e Investigación. 2002; 5(1): 23-29.

14. Svobodová E, et al. Genetic diversity of yacon (Smallanthus sonchifolius (Poepp. \& Endl.) H. Robinson) and its wild relatives as revealed by ISSR markers. Biochemical systematics and ecology. 2013; 50: 383-389.

15. Ojansivu I, Ferreira CL, Salminen S. Yacon, a new source of prebiotic oligosaccharides with a history of safe use. Trends in Food Science \& Technology. 2011; 22(1): 40-46.

16. Arnao A.l, et al. Efecto hepatoprotector del extracto acuoso de Smallanthus sonchifolius (yacón) en un modelo de intoxicación con acetaminofén. in Anales de la Facultad de Medicina. UNMSM. Facultad de Medicina. 2012:239-244

17. Hernández J, León J. Cultivos marginados: otra perspectiva de 1492. 1992.

18. Jiménez Rodríguez K.M. Propuesta para el cultivo y aprovechamiento sostenible del yacón (Smallanthus sonchifolius (Poepp. y Endl.) H. Rob) en Colombia. Bogotá D.C Colombia: Universidad Pontificia Javeriana Facultad de Ciencias Carrera de Biología, 2011.

19. Rajchl A, et al., Characterisation of yacon tuberous roots and leaves by DARTTOF/MS. International Journal of Mass Spectrometry. 2018; 424: 27-34.

20. Oliveira RB, et al. Topical anti-inflammatory activity of yacon leaf extracts. Revista Brasileira de Farmacognosia. 2013; 23(3): 497-505.

21. De Moura NA, et al. Protective effects of yacon (Smallanthus sonchifolius) intake on experimental colon carcinogenesis. Food and Chemical Toxicology. 2012; 50(8): 2902-2910.
22. Sumiyanto J, et al. Oligofructans content and yield of yacon (Smallanthus sonchifolius) cultivated in Mississippi. Scientia horticulturae. 2012; 148: 83-88.

23. Muñoz A.M, et al. Evaluación del contenido nutricional de yacón (Polimnia sonchifolia) procedente de sus principales zonas de producción nacional. Horizonte Médico. 2006. 6(2).

24. Mansilla R, et al. Estudios de la biología reproductiva en cinco accesiones de Smallanthus sonchifolius (Poepp. \& Endl.) Robinson. Ecología Aplicada. 2010; 9(2): 167-175.

25. Chasquibol $\mathrm{N}$, et al. Estudio químico y nutricional de las variedades de la raíz de la Polymnia sonchifolia "YACÓN". Revista Peruana de Química e Ingeniería Química. $2002 ; 5(1): 37-42$.

26. Sousa $S$, et al. In vitro evaluation of yacon (Smallanthus sonchifolius) tuber flour prebiotic potential. Food and Bioproducts Processing. 2015; 95: 96-105.

27. Roberto $C$, et al. Analisis de la variabilidad molecular de una colección peruana de Smallanthus sonchofolius (Poepp \& Endl) H. Robinson. Ecología Aplicada. 2006; 5(1, 2): 75-80.

28. Simonovska B, et al. Investigation of phenolic acids in yacon (Smallanthus sonchifolius) leaves and tubers. Journal of chromatography A. 2003; 1016 (1): 89-98.

29. Da Silva, MFGD., et al. Evaluation of nutritional and chemical composition of yacon syrup using $1 \mathrm{H}$ NMR and UPLCESI-Q-TOF-MS E. Food Chemistry, 2017.

30. Mayta $P$, et al. Reducción de la respuesta glicémica posprandial post-ingesta de raíz fresca de yacón en sujetos sanos. CIMEL Ciencia e Investigación Médica Estudiantil Latinoamericana. 2004; 9(1).

31. Genta S, et al. Yacon syrup: beneficial effects on obesity and insulin resis- 
tance in humans. Clinical Nutrition. 2009; 28(2):182-187.

32. Paredes LL, et al. Yacon fructans (Smallanthus sonchifolius) extraction, characterization and activation of macrophages to phagocyte yeast cells. International journal of biological macromolecules, 2017.

33. Morales IJL, Acevedo JLA, Hilario CBC. Actividad antioxidante in vitro del extracto acuoso de las hojas de Smallantus sonchifolius (yacón). Conocimiento para el desarrollo. 2016; 7(2).

34. Grancieri M. et al. Yacon flour (Smallanthus sonchifolius) attenuates intestinal morbidity in rats with colon cancer. Journal of Functional Foods. 2017; 37: 666-675.

35. Muñoz AM, et al. Evaluación de la capacidad antioxidante y contenido de compuestos fenólicos en recursos vegetales promisorios. Revista de la Sociedad Química del Perú. 2007; 73(3):142-149.

36. Lillo M, De Tucumán S. Morfología y anatomía foliar de "yacón", Smallanthus sonchifolius (Asteraceae), con fines de control de calidad. Acta farmacéutica bonaerense. 2006; 25(4): 526-32.

37. Aguilar E, Bonilla P. Actividad antioxidante e inmunológica de flavonoides aislados de hojas de Smallanthus sonchifolius (yacón). Ciencia e Investigación. 2009;12(1): 15-23.

38. Franco TS, et al. Foam mat drying of yacon juice: Experimental analysis and computer simulation. Journal of Food Engineering 2015;158: 48-57.

39. Campos, D., et al., Prebiotic effects of yacon (Smallanthus sonchifolius Poepp. \& Endl), a source of fructooligosaccharides and phenolic compounds with antioxidant activity. Food Chemistry, 2012. 135(3): p. 1592-1599.

40. Grau A, et al. El yacón como alimento, fuente de suplementos dietarios y de productos farmacéuticos: un panorama histórico, el presente y el futuro. Boletín Latinoamericano y del Caribe de Plantas Medicinales y Aromáticas. 2007; 6(5): 173-174.

41. Valentová K, et al. Maca (Lepidium meyenii) and yacon (Smallanthus sonchifolius) in combination with silymarin as food supplements: in vivo safety assessment. Food and Chemical toxicology. 2008;46(3):1006-1013.

42. Baroni S, et al. Hydroethanolic extract of Smallanthus sonchifolius leaves improves hyperglycemia of streptozotocin induced neonatal diabetic rats. Asian Pacific journal of tropical medicine. 2016; 9(5): 432-436.

43. Genta S.B, et al. Hypoglycemic activity of leaf organic extracts from Smallanthus sonchifolius: constituents of the most active fractions. Chemico-Biological Interactions. 2010;185(2): 143-152.

44. Honoré S.M, et al. Protective effect of yacon leaves decoction against early nephropathy in experimental diabetic rats. Food and Chemical Toxicology. 2012; 50(5):1704-1715.

45. Mentreddy S, Mohamed A, Rimando A. Medicinal plants with hypoglycemic/ anti-hyperglycemic properties: a review. in Proc Assoc Adv Ind Crop Conf. 2005 p.341-343

46. Sánchez S, Genta S. Yacón: un potencial producto natural para el tratamiento de la diabetes. Boletín Latinoamericano y del Caribe de Plantas Medicinales y Aromáticas. 2007; 6(5):162-164.

47. Da Silva M.D.F.G, et al. Yacon syrup: Food applications and impact on satiety in healthy volunteers. Food Research International. 2017. 100:460-467.

48. Dionísio A.P, et al. Cashew-apple (Anacardium occidentale L.) and yacon (Smallanthus sonchifolius) functional beverage improve the diabetic state in rats. 
Food Research International. 2015; 77 : 171-176.

49. Parussolo G, et al. Synbiotic ice cream containing yacon flour and Lactobacillus acidophylus NCFM. LWT. Food Science and Technology. 2017; 82:192-198.

50. Das Graças Vaz-Tostes M, et al. Yacon effects in immune response and nutritional status of iron and zinc in preschool children. Nutrition. 2014; 30(6):666-672.

51. Sousa S, et al., Antioxidant properties of sterilized yacon (Smallanthus sonchi- folius) tuber flour. Food chemistry. 2015; 188: 504-509.

52. Zamora S. Antioxidantes: Micronutrientes en lucha por la salud. Revista chilena de nutrición. 2007; 34:17-26.

53. De Oliveira RB, et al. Renal toxicity caused by oral use of medicinal plants: the yacon example. Journal of ethnopharmacology. 2011; 133(2): 434-441. 
